\title{
Exploring the Likely Effect of the Introduction of Drug Eluting Stents on Requirements for Coronary Artery Revascularisation Procedures in Western Australia: A Use of the CHD/CARP Markov Simulation Model
}

\author{
Haider R Mannan ${ }^{*}, 1,2$ and Matthew Knuiman ${ }^{2}$ \\ ${ }^{I}$ Department of Epidemiology \& Preventive Medicine, School of Public Health \& Preventive Medicine, Monash Univer- \\ sity, Alfred Campus, Melbourne, Victoria 3004, Australia \\ ${ }^{2}$ School of Population Health, University of Western Australia, Perth, WA, Australia
}

\begin{abstract}
Background: Coronary artery revascularisation procedures (CARPs) include coronary artery bypass graft procedures (CABGs) and the less invasive percutaneous coronary interventions (PCIs) and they are common surgical interventions for coronary heart disease (CHD). The effectiveness of PCIs increased when stents were introduced and there was also a shift towards doing more PCIs and less CABGs, especially in older patients. More recently, PCIs have been further improved by the use of drug-eluting-stents (DES). In this study we used a recently developed CHD/CARP Markov model to explore the likely effect on CARP requirements due to the introduction of DES.

Methods: This is achieved by considering the population of Western Australia aged 35 to 79 years at the beginning of 2001 (grouped according to history of CHD and CARPs) as the cohort, calculating the mean population risks for CHD/CARP events over three years 1998 to 2000, and using these population risks and certain modifications of them that incorporate the likely effect of the introduction of DES (in 2002) in the CHD/CARP Markov model to explore difference in total requirements for CABGs and PCIs over the period 2001 to 2010 . The anticipated likely effect of DES on probabilities of CARP procedures was based on results of meta-analysis of randomized controlled trials comparing DES stents to ordinary stents and an anticipated further reduction in CABGs and increase in PCIs as was observed when (ordinary) stents were introduced.
\end{abstract}

Results: The simulation results suggest that, over the period 2001 to 2010 , the total number of CABGs will decline by up to $19 \%$ and the total number of PCIs will increase by up to $6 \%$.

Conclusion: The introduction of DES will have greater effect on the requirement of CABGs as compared to that on PCIs.

\section{INTRODUCTION}

Although rates of coronary heart disease (CHD) have been declining for 20 years it still remains a major cause of morbidity and mortality [1]. Surgical interventions for CHD include coronary artery bypass graft procedures (CABGs) and the less invasive percutaneous coronary interventions (PCIs) and together these are known as coronary artery revascularisation procedures (CARPs). The effectiveness and scope of PCI procedures increased, first with the development of balloon angioplasty, then with the insertion of stents to keep the coronary arteries open, and more recently with the use of drug-eluting stents (DES). Meta-analyses of randomized controlled trials [2] and population based observational studies [3] have already shown that ordinary stents have contributed to a decline in the risk of a repeat CARP. More recent meta-analyses of randomized controlled trials comparing drug-eluting to ordinary stents [4-7] suggest even greater effectiveness. Moreover, a population based modeling approach has suggested that DES will lead to a further reduction in the need for revision procedures [3].

*Address correspondence to this author at the Department of Epidemiology $\&$ Preventive Medicine, School of Public Health \& Preventive Medicine, Monash University, Alfred Campus, Melbourne, Victoria 3004, Australia; E-mail: haider.mannan@med.monash.edu.au
In Australia, CARPs are common as illustrated by agestandardised rates for PCI and CABG of 1,022 and 809 per million population in the year 2000 [8]. The state of Western Australia has seen very rapid uptake of PCI technology, with PCIs making up 64 percent of all CARPs during 2000-2001 [9]. Ordinary stents were introduced into PCI procedures in 1995 with $90.7 \%$ of PCI procedures using stents by 2000 2001. DES were introduced in 2002 and by 2005 about $90 \%$ of PCIs performed in Western Australia were using DES [10]. The uptake however has not been as rapid in other states of Australia [11, 12].

The introduction of stents had two principal effects on CARP requirements. First, it reduced the risk of a repeat CARP following a primary PCI and second, it initiated a shift towards doing less CABGs and more PCIs. Randomised control trial evidence indicates that the introduction of DES will reduce the risk of a repeat CARP following primary PCI even further $[6,7]$ but it is unclear if it will initiate a further shift from CABG to PCI overall.

In view of the rapid uptake of DES and the uncertainty about what the effect of DES might be on the requirements of CARPs, we have used a recently developed CHD/CARP Markov simulation model [13-15] to explore the likely effects of DES on future requirements for CARPs in the Western Australian population. 


\section{METHODS}

Models: The CHD/CARP Markov simulation model has been described in detail previously [13-15]. Briefly, the model is based on annual risks, stratified by age, gender and CHD/CARP history, for admission to hospital for CHD, for a PCI, for a CABG, for CHD death and non-CHD death. Each member of a population cohort is placed in one of several mutually exclusive CHD/CARP history states based on their hospital admissions history for the period 1980 to 2000: [1] a history of having a PCI but no CABG some time in the past; [2] a history of having a CABG some time in the past; [3] a history of having a CHD admission but no CABG or PCI some time in the past; or [4] no history of CHD admission, $\mathrm{CABG}$ and $\mathrm{PCI}$ in the past. The model allows members to move from one state to another in each cycle (year) according to the events they experience during the year. Within a cycle, the model allows for a CHD admission without a CARP, up to two admissions with CARPs (CABG or PCI) in addition to possible death. The schematic diagram for the model is shown in Fig. (1).

Data: The model is applied to the cohort comprising the population of the state of Western Australia aged 35 to 79 years at the beginning of 2001. Using linked records for individuals in the WA Health Linked Data system [16] together with population census data, each member of the Western Australian population cohort is placed in one of the four hospital admissions history states.

Analysis: The model incorporates ageing of cohort members and requires values of event risks for each age, gender and history state for each year in order for the events for the cohort to be simulated. In the context of forecasting, population event risks for past years (1998 to 2000 in our case) are calculated and used to project event risks for future years. The validation of the model and a demonstration of its use for forecasting (from 1995 to 1999) have been described and discussed previously [13-15].
To simulate the numbers of CABGs and PCIs for the 2001 WA population cohort over the period 2001 to 2010, the average population event risks for 1998 to 2000 are used for each year from 2001 to 2010 . To assess the likely effect of the introduction of stents in 2002, some of the CARP event risks are altered for the years 2002 to 2010 . The results reported in this paper are based on the assumption that all patients undergoing a PCI in 2002 and onwards receive a DES. This is reasonable considering that, in Western Australia, about 90 percent of all patients undergoing a PCI have been getting a DES since its introduction [10].

Two types of event risks are altered due to the introduction of DES. First, the risks of a second PCI and/or CABG in the same year following a primary PCI are altered to reflect the increased effectiveness of DES over ordinary stents. These repeat CARP risks were observed to decline after the introduction of ordinary stents in 1995 and the relative risk reduction at the population level which resulted from the introduction of ordinary stents in 1995 was similar to that found in meta-analyses of randomized controlled trials [9, 14]. This is the basis for altering these same event risks following introduction of DES. The evidence from several meta-analyses of randomized controlled trials that compare DES to ordinary stents is that the use of DES will reduce the risk of restenosis and CABG by about $40 \%$ [4-6] while another meta-analysis has shown DESs to reduce the risk of repeat PCI by about 79 percent and CABG by 69 percent [7]. In our simulation we have used a risk reduction for repeat CARP (when the first CARP is PCI) of $50 \%$.

The second type of event risk that was altered was the overall risk of a PCI and a CABG. It was observed that for the period 1995-99 the risk of a PCI increased while the risk of a CABG declined after the introduction of ordinary stents in $1995[14,15]$. Thus, we consider another scenario that the introduction of DES will result in a further increase in the risk a PCI and a decrease in the risk of a CABG. These changes in risk are expected to capture a further shift

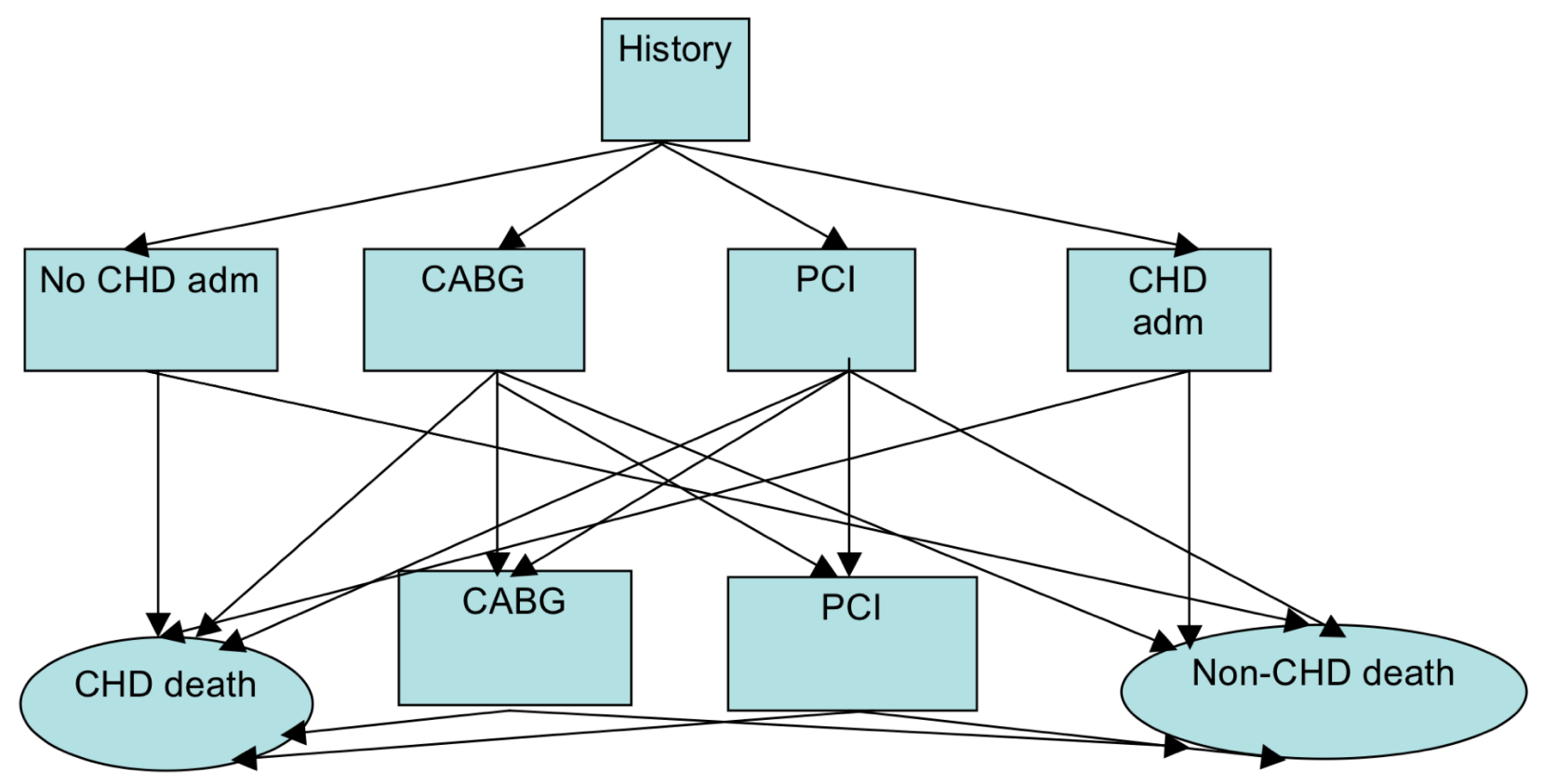

Fig. (1). Schematic diagram for the recognised events during a cycle (year). 
towards more PCIs and less CABGs. The risk changes following introduction of stents in 1995 was a $25 \%$ increase in PCI and a $15 \%$ decrease in risk of CABG for males and a $12.5 \%$ increase in risk of PCI and $7.5 \%$ decrease in risk of $\mathrm{CABG}$ for females. Based on these values and expert opinion we assume that the effect of introduction of DES will be to increase risk of PCI by $10 \%$ and reduce the risk of CABG by $10 \%$.

Three simulations are conducted [1] using the average events risks from 1998-2000 for all event risks [2] using the average event risks from 1998-2000 for all events except the risk for repeat CARPs when the primary CARP is PCI which are modified as described above, and [3] using the average event risks from 1998-2000 for all events except for the risk of repeat CARPs when the primary CARP is PCI and the overall risk of CABG and PCI which are modified as described above. These three simulation scenarios are called Mean, DES [1] and DES [2] respectively.

\section{RESULTS}

Table 1 shows the total simulated number of CABGs and PCIs over the period 2001-2010 under the three sets of event risk described in Methods for the population of Western Australia aged 35-79 years in $2001(n=8,97,477)$. In comparison to the Mean method, the total number of CABGs decreased by 7.4 percent under DES [1] and by 19.2 percent under DES(2). The total number of PCIs decreased by 3.4 percent under DES [1] and increased by 6.3 percent under DES [2].

Since the effect of DES on future numbers of CARPs may take a few years to emerge, it is important to look at predicted event numbers by each calendar year. Fig. (2) shows the total projected numbers of CABGs and PCIs obtained under the three scenarios by single year over 20012010. This figure shows that differences increase slowly each year from 2003 .
Table 1. Comparison between Projected Numbers of CARPs (2001-2010) Based on Mean, DES(1) and DES(2) Scenarios for Western Australia Population 35-79 Years in 2001

\begin{tabular}{|c|c|c|}
\hline Scenario & Total CABGs 2001-2010 & Total PCIs 2001-2010 \\
\hline \hline Mean & 15,752 & 24,474 \\
\hline DES(1) & $14,588(-7.4 \%)$ & $23,631(-3.4 \%)$ \\
\hline DES(2) & $12,728(-19.2 \%)$ & $26,025(+6.3 \%)$ \\
\hline
\end{tabular}

\section{DISCUSSION}

The forecasts regarding future requirements for CABGs and PCIs as performed in this study were based on reasonably conservative estimates of the effect of DES on repeat procedures and the shift from CABGs to PCIs. The modeling of 10 percent magnitude of change in the risk of a $\mathrm{CABG}$ and that of a PCI for the final DES scenario deserves some explanation. Based on expert opinion we assumed that the magnitude of these changes were not as pronounced when DES was introduced compared to ordinary stent. The magnitude of 10 percent change in the relevant risks since 2002 is almost half the change in these risks which occurred after ordinary stents were introduced in WA in 1995 [9] and hence we believe that the scenario we developed was fairly reasonable. We simulated another DES scenario with a magnitude of 20 percent change in the relevant risks and also found that the introduction of DES will result in a smaller increase in the requirements for PCIs but a larger decrease in the requirements for CABGs (data not given).

Thus, all the forecasts regarding future trends for CABGs and PCIs as performed in this study are expected to be reasonable as we believe that we did not seriously over- or under- estimate the magnitude of the changes in the transition

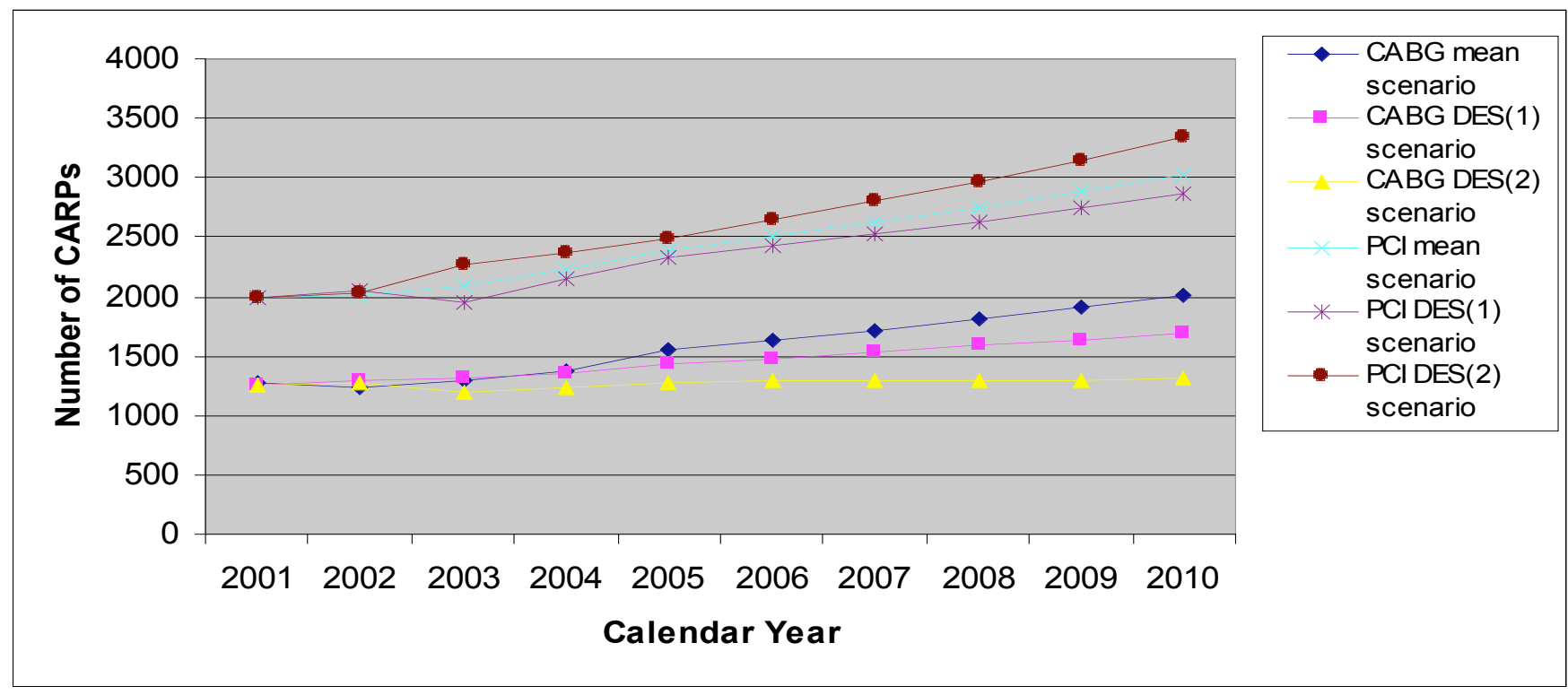

Fig. (2). Comparison between total projected numbers of CABGs and PCIs each year under mean, DES(1) and DES(2) scenarios over the period 2001-2010. 
probabilities at the population level based on results from meta-analyses of RCTs and was able to foresee major future changes in the model's parameters due to advancement in PCIs. Thus, these forecasts represent our best projections based on the premise that the events of the recent past can be used as a guide to the future.

The single year projections showed that the effect of DES on the requirements for CARPs gradually increases with time. When the increased effectiveness of PCI due to an introduction of DES was captured by reducing the risks of a repeat PCI (DES [1]), there was a small decline in the requirements for PCIs with the effect having a lag and a larger decline in the requirements for CABGs. When further changes were incorporated that captured a shift in practice between a CABG and a PCI (DES [2]) there was a small increase in the requirements for PCIs with a lag in the effect but a substantial decrease in the requirements for CABGs.

For simplicity, we have incorporated the effect of DESs into the projected risks by a constant relative risk reduction for the specified events. However, whilst this is reasonable for Western Australia where the uptake is very rapid [10], the uptake of DES in other populations may be more gradual. The CHD/CARP simulation model is flexible enough to model this. Also, for simplicity we have assumed that the decline in these risks is uniform across both sexes and all age groups. It is possible that the DES effect may vary by age and sex. We did not however find any evidence in support of this as no meta-analysis has examined the effect of DES on these outcomes in different age-sex subgroups of patients.

In Australia, the cost of DESs is about three to four times that of ordinary stents [12]. As a result, DES use in the public health system (except in WA) is reserved for selected cases (eg, for patients at high risk of restenosis) [12]. However, unrestricted DES use as in WA may not be economically viable under the public health system. A recent costeffectiveness analysis in the US suggested the sirolimuseluting stent would be a cost effective treatment strategy when the rate of restenosis exceeds $18.5 \%$ [17]. A recent Australian study showed that limiting DES use to patients at the highest risk of restenosis might improve the costeffectiveness of DESs in an Australian model based on randomised trial results [6].

The CHD/CARP model can also be used to determine the effect on requirements of CARPs of advances in medical treatments related to CHD, changes in trends of CHD incidence stemming from changes in risk factor trends (eg, the obesity epidemic) and changes in health policy related to treating CHD patients. Examples of determining effects of advances in medical treatments include increasing use of prescribed aspirin, lipid lowering drugs, statins and ACE inhibitors. Examples of examining effects on requirements of CARPs due to changes in health policy include change in health policy (by the Australian government) regarding subsidising statins by only providing subsidy to people at higher risk of CHD. In terms of the CHD/CARP model, this can be explored by altering incidence transition probabilities to take into consideration more restricted access to subsidized statins. The CHD/CARP model can further be used to determine the cost of future requirements for CARPs and to explore the most cost-effective strategies for treatment of CHD. To achieve this indicative cost estimates are needed for each of the alternative CARP treatments including CABG and PCI with ordinary stent and DES. Such cost data on alternative CARP treatments may be obtained from information from the patient costing databases at the WA teaching hospitals. Future cost estimates may be obtained by time series modelling using information relating to variables such as Commonwealth Medicare Benefit Schedule (CMBS) rates, Diagnosis Related Group (DRG) costs, procedure-specific costs, theatre time and lengths of hospital stay. Expert advice regarding costs can also be obtained from clinicians and hospital finance personnel.

\section{REFERENCES}

[1] Uemara K, Pisa Z. Trends in cardiovascular disease mortality in industrialized countries since 1950. World Health Stat Q Rep 1988; 41: 155-78.

[2] Brophy JM, Belisle P, Joseph L. Evidence of use of coronary stents: a hierarchical Bayesian meta-analysis. Ann Intern Med 2003; 138: 777-86.

[3] McCaul KA, Hobbs MST, Knuimam MW, et al. Trends in two year risk of repeat revascularisation or death from cardiovascular disease after coronary artery bypass grafting or percutaneous coronary intervention in Western Australia, 1980-2001. Heart 2004; 90: 1042-6.

[4] Katritsis DG, Karvouni E, Loannidis JPA. Meta-analysis comparing drug-eluting stents with bare metal stents. Am J Cardiol 2005; 95: 640-3.

[5] Babapulle MN, Joseph L, Belisle P, Brophy JM, Eisenberg MJ. A hierarchical Bayesian meta-analysis of randomized clinical trials of drug-eluting stents. Lancet 2004; 364: 583-91.

[6] Lord SJ, Howard K, Allen F, et al. A systematic review and economic analysis of drug-eluting coronary stents available in Australia. Med J Aust 2005; 183: 464-71.

[7] Sondhi M, Jagannath A, Wong JB. A meta-analysis of randomized controlled trials with coronary drug-eluting stents. Poster presented in the $26^{\text {th }}$ Annual Meeting of the Society for Medical Decision Making, October 17-20, 2004.

[8] AIHW (Australian Institute of Health and Welfare): 2003. Coronary revascularization in Australia 2000; Bulletin, Issue 7.

[9] Hobbs MST, McCaul KA, Knuiman MW, et al. Trends in coronary artery revascularization procedures in Western Australia, 19802001. Heart 2004; 90: 1036-41.

[10] Hobbs MST, Professor Emeritus. School of Population Health, University of Western Australia, Perth, WA, Australia, personal communication 2006.

[11] Rankin J. Interventional Cardiologist, Royal Perth Hospital, Perth, WA; Chew DP, Interventional Cardiologist, Flinders Medical Centre, Bedford Park SA, Muller DM. Interventional Cardiologist, Director of Cardiac Catheterisation Laboratory, St Vincent's Hospital, Darlinghurst, NSW; personal communications, March 2006.

[12] Yan BP, Ajani AE, Duffy SJ, et al. Use of drug-eluting stents in Victorian public hospitals. Med J Aust 2006; 185: 363-7.

[13] Mannan HR. Development and use of a Monte Carlo-Markov cycle tree model for coronary heart disease incidence/mortality and health service usage with explicit recognition of coronary artery revascularization procedures (CARPs). Unpublished Ph.d dissertation. University of Western Australia, Perth, Australia 2007.

[14] Mannan HR, Knuiman MK, Hobbs M. A Markov simulation model for analysing and forecasting the number of coronary artery revascularization procedures in Western Australia. Ann Epidemiol 2007; 17: 964-75.

[15] Mannan HR, Knuiman MK, Hobbs M. Adapting a Markov Monte Carlo simulation model for forecasting the number of Coronary Artery revascularisation procedures in an era of rapidly changing technology and policy. BMC Med Inform Decis Mak 2008; 8: 27. 
[16] Holman CD, Bass AJ, Rouse IL, et al. Population-based linkage of health records in Western Australia: development of a health services research linked database. Aust New Zeal J Publ Health 1999; 23: 453-9.

[17] Cohen DJ, Bakhai A, Shi C, et al. SIRIUS Investigators. Costeffectiveness of sirolimus-eluting stents for treatment of complex coronary stenoses: results from the sirolimus-eluting balloon expandable stent in the treatment of patients with de novo native coronary artery lesions (SIRIUS) trial. Circulation 2004; 110: 50814.

(C) Mannan and Knuiman; Licensee Bentham Open.

This is an open access article licensed under the terms of the Creative Commons Attribution Non-Commercial License (http://creativecommons.org/licenses/bync/3.0/) which permits unrestricted, non-commercial use, distribution and reproduction in any medium, provided the work is properly cited. 